\title{
Pacific
}

Journal of

Mathematics

\section{ON SLIM DOUBLE LIE GROUPOIDS}

Nicolas Andruskiewitsch, Jesus OchoA ARANGO AND AlEJandro TIRABOSCHI 


\title{
ON SLIM DOUBLE LIE GROUPOIDS
}

\author{
Nicolas AndruskiEwitsch, Jesus OCHOA ARANGO \\ AND ALEJANDRO TIRABOSCHI
}

\begin{abstract}
We prove that every slim double Lie groupoid with proper core action is completely determined by a factorization of a canonically defined "diagonal" Lie groupoid.
\end{abstract}

\section{Introduction}

A double groupoid is a set $\mathscr{B}$ provided with two different but compatible groupoid structures. It is useful to represent the elements of $\mathscr{B}$ as boxes that merge horizontally or vertically according to the groupoid multiplication into consideration. The vertical sides of a box belong to a groupoid $\mathscr{V}$, and the horizontal sides to a groupoid $\mathscr{H}$. A double groupoid is slim if any box is determined by its four sides. The notion of double groupoids was introduced by Ehresmann [1963], and later studied in [Brown 2004; Brown and Janelidze 2004; Brown and Mackenzie 1992; Brown and Spencer 1976] and references therein.

The notion of double Lie groupoid was defined and investigated by K. Mackenzie [1992; 2000]; see also [Pradines 1977; Mackenzie 1999; Lu and Weinstein 1989] for applications to differential and Poisson geometry. In particular the question of the classification of double Lie groupoids was raised in [Mackenzie 1992]; see also [Brown and Mackenzie 1992]. In the latter article, a complete answer was given in the restricted case of locally trivial double Lie groupoids. More recently, a description in two stages of discrete double groupoids was given in [Andruskiewitsch and Natale 2009]. To state them, let us recall that a diagram over a pair of groupoids $\mathscr{V}$ and $\mathscr{H}$ is a triple $(\mathscr{D}, j, i)$ where $\mathscr{D}$ is a groupoid and $i: \mathscr{H} \rightarrow \mathscr{D}, j: \mathscr{V} \rightarrow \mathscr{D}$ are morphisms of groupoids (over a fixed set of points). The stages are:

(a) Any double groupoid is an extension of slim double groupoid (its frame) by an abelian group bundle.

This work was partially supported by Pontificia Universidad Javeriana, Bogotá, Colombia; CONICET, Fundación Antorchas, Agencia Córdoba Ciencia, ANPCyT and Secyt (UNC) (Argentina). MSC2010: 18D05, 20L05, 22A22.

Keywords: Lie groupoid, core groupoid, proper action, double groupoid, core action, groupoid diagram. 
(b) The category of slim double groupoids, with fixed vertical and horizontal groupoids $\mathscr{V}$ and $\mathscr{H}$, satisfying the filling condition, is equivalent to the category of diagrams over $\mathscr{V}$ and $\mathscr{H}$.

In this paper, we extend stage (b) to the setting of double Lie groupoids. In this context the top-right map has to be a surjective submersion, a differentiable version of the filling condition [Mackenzie 1992]. As one may expect, there are some topological and geometrical ingredients in our main result, which is this:

Theorem 3.7. The category of slim double Lie groupoids, with fixed vertical and horizontal Lie groupoids $\mathcal{V}$ and $\mathcal{H}$, and proper core action, is equivalent to the category of diagrams of Lie groupoids $(\mathscr{D}, j, i)$ such that the maps $j$ and $i$ are transversal at the identities.

Our proof of this theorem relies on [Andruskiewitsch and Natale 2009, Theorem 2.8 ] and some topological and differentiable considerations. We should note that the properness of the core action really boils down to the closedness of the anchor map of the corresponding action groupoid, since the core action is actually free.

We have started to investigate the stage (a) of the above construction in the differentiable setting; we expect to present this analysis in a future paper.

\section{Preliminaries on Lie groupoids an double Lie groupoids}

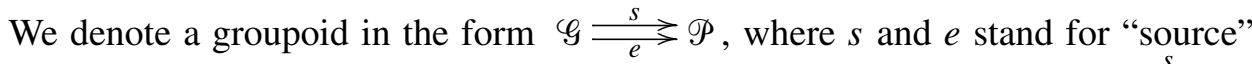

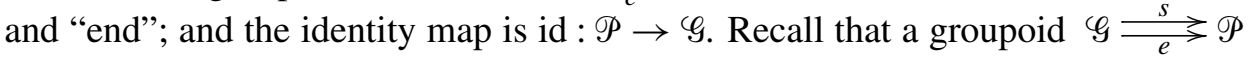
is a Lie groupoid [Mackenzie 2005], if $\mathscr{P}$ and $\mathscr{G}$ are smooth manifolds, $s$ and $e$ are surjective submersions and the other structural maps are smooth. The anchor of $\varphi$ is the map $\chi: \mathscr{G} \rightarrow \mathscr{P} \times \mathscr{P}$ given by $\chi(g)=(s(g), e(g))$.

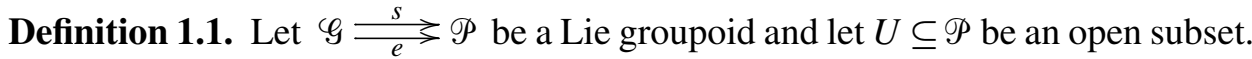
A local bisection of $\mathscr{G}$ on $U$ is a smooth map $\sigma: U \rightarrow \mathscr{G}$ which is a section of $e$ and such that $V:=(s \circ \sigma)(U)$ is an open subset of $\mathscr{P}$ and $s \circ \sigma: U \rightarrow V$ is a diffeomorphism. Define $\varphi^{U}=s^{-1}(U)$ and $\mathscr{G}_{U}=e^{-1}(U)$; the local left and right translations induced by $\sigma$ are the maps

$$
\begin{array}{ll}
L_{\sigma}: \mathscr{G}^{U} \rightarrow \mathscr{G}^{V}, & g \mapsto \sigma(s(g)) g ; \\
R_{\sigma}: \mathscr{G}_{V} \rightarrow \mathscr{G}_{U}, & g \mapsto g \sigma\left((s \circ \sigma)^{-1}(e(g))\right) .
\end{array}
$$

For more on bisections see [Mackenzie 2005, Section 1.4].

\section{Double Lie groupoids.}

Definition 1.2 (Ehresmann). A double groupoid is a groupoid object internal to the category of groupoids. In other terms, a double groupoid consists of a set $\mathscr{B}$ with two groupoid structures with bases $\mathscr{H}$ and $\mathscr{V}$, which are themselves groupoids 
over a common base $\mathscr{P}$, all subject to the compatibility condition that the structure maps of each structure are morphisms with respect to the other.

It is usual to represent a double groupoid $(\mathscr{B} ; \mathscr{V}, \mathscr{H} ; \mathscr{P})$ as a diagram of four related groupoids

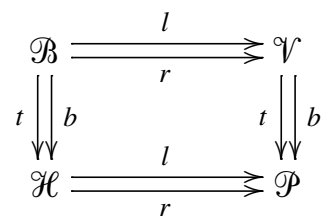

where $t, b, l, r$ mean "top", "bottom", "left" and "right". We sketch the main axioms that these groupoids should satisfy and refer to, for instance, [Andruskiewitsch and Natale 2005, Section 2] and [Andruskiewitsch and Natale 2006, Section 1] for detailed exposition and other conventions.

The elements of $\mathscr{B}$ are called boxes and will be denoted by

$$
A=l(A) \underbrace{t(A)}_{b(A)} A \quad r(A) \in \mathscr{B} .
$$

Here $t(A), b(A) \in \mathscr{H}$ and $l(A), r(A) \in \mathscr{V}$. The identity maps will be denoted id $: \mathscr{V} \rightarrow \mathscr{B}$ and id $: \mathscr{H} \rightarrow \mathscr{B}$. The product in the groupoid $\mathscr{B}$ with base $\mathscr{V}$ is called horizontal and denoted by $A B$ or $\{A B\}$, for $A, B \in \mathscr{B}$ with $r(A)=l(B)$. The product in the groupoid $\mathscr{B}$ with base $\mathscr{H}$ is called vertical and we shall denote it by ${ }_{B}^{A}\left\{\begin{array}{l}A \\ B\end{array}\right\}$, for $A, B \in \mathscr{B}$ with $b(A)=t(B)$. This pictorial notation is useful for understanding products in the double structure. For instance, the compatibility axioms between the horizontal and vertical products are described by
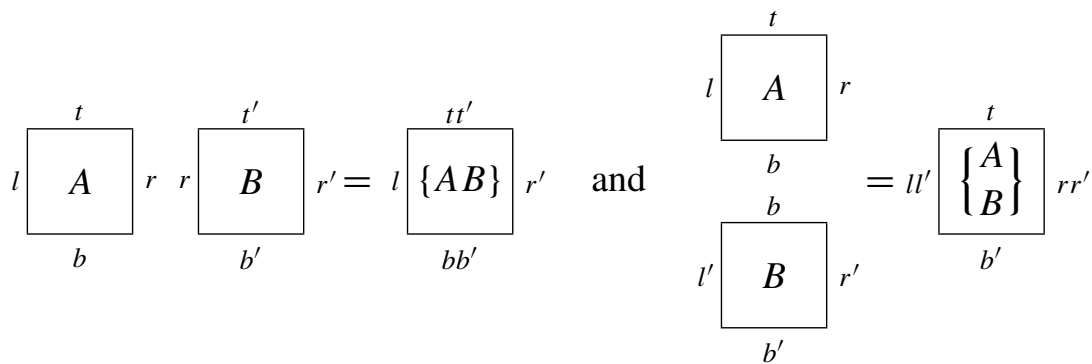

We omit the letter inside the box if no confusion arises. We also write $A^{h}$ and $A^{v}$ to denote the inverse of $A \in \mathscr{B}$ with respect to the horizontal and vertical structures of groupoid over $\mathscr{B}$ respectively. When one of the sides of a box is an identity, we draw this side as a double edge. For example, if $t(A)=\mathrm{id}_{p}$, we draw $\square$ and say that $t(A) \in \mathscr{P}$. 
Definition 1.3 [Mackenzie 1992]. A double groupoid is a double Lie groupoid if all the four groupoids involved are Lie groupoids and the top-right corner map

$$
\urcorner: \mathscr{B} \rightarrow \mathscr{H}_{l} \times_{t} \mathscr{V}, \quad A \mapsto\right\urcorner(A)=(t(A), r(A)),
$$

is a surjective submersion.

We shall say that a double groupoid is discrete if no differentiable or topological structure is present. A discrete double groupoid satisfies the filling condition if the top-right corner map defined in (1-2) is surjective. We refer the reader to [Andruskiewitsch and Natale 2009] for more details on discrete double groupoids and the filling condition.

Definition 1.4 [Brown and Mackenzie 1992; Mackenzie 1992]. Let $(\mathscr{B} ; \mathscr{V}, \mathscr{H} ; \mathscr{P})$ be a double Lie groupoid. The core groupoid $\mathbf{E}(\mathscr{B})$ of $\mathscr{B}$ is the set

$$
\mathbf{E}(\mathscr{B})=\{E \in \mathscr{B}: t(E), r(E) \in \mathscr{P}\}=(\urcorner)^{-1}\left\{\left(\operatorname{id}_{p}, \mathrm{id}_{p}\right): p \in \mathscr{P}\right\}
$$

with source and target projections $s_{\mathbf{E}}, e_{\mathbf{E}}: \mathbf{E}(\mathscr{B}) \rightarrow \mathscr{P}$, given by $s_{\mathbf{E}}(E)=b l(E)$ and $e_{\mathbf{E}}(E)=\operatorname{tr}(E)$ respectively; identity map given by $\operatorname{id}_{p}=\Theta_{p}:=\mathbf{i d} \circ \operatorname{id}(p)$; multiplication and inverse given by

$(1-3) \quad E \circ F:=\left\{\begin{array}{cc}\operatorname{id} l(F) & F \\ E & \operatorname{id}(b(F))\end{array}\right\}, \quad E^{(-1)}:=\left(E \operatorname{id} b(E)^{-1}\right)^{v}=\left\{\begin{array}{c}\mathbf{i d} l(E)^{-1} \\ E^{h}\end{array}\right\}$,

for every compatible $E, F \in \mathbf{E}(\mathscr{B})$.

We observe that the elements of $\mathbf{E}(\mathscr{B})$ are of the form $E=\square$; the source gives the bottom-left vertex and the target gives the top-right vertex of the box. Since the top-right corner map is a surjective submersion, the core groupoid $\mathbf{E}(\mathscr{B})$ is a closed embedded submanifold of $\mathscr{R}$ and clearly $s_{\mathbf{E}}$ and $e_{\mathbf{E}}$ are surjective submersions. With these structural maps $\mathbf{E}(\mathscr{B})$ becomes a Lie groupoid with base $\mathscr{P}$ (differentiability conditions being easily verified because $\mathbf{E}(\mathscr{B})$ is an embedded submanifold of $\mathscr{B}$ ).

Coarse double groupoid. Let $\mathscr{P}$ be a set and $\mathscr{V}$, $\mathscr{H}$ be groupoids over $\mathscr{P}$. Let $\square(\mathcal{V}, \mathscr{H})$ be the set

$$
\left(\mathscr{V}_{b} \times_{l} \mathscr{H}\right)_{(t, r)} \times_{(l, b)}\left(\mathscr{H}_{t} \times_{r} \mathscr{V}\right)
$$

that is, the set of quadruples $\left(\begin{array}{c}x \\ f_{y} g\end{array}\right)$ with $x, y \in \mathscr{H}, f, g \in \mathscr{V}$ such that

$$
l(x)=t(f), \quad r(x)=t(g), \quad l(y)=b(f), \quad r(y)=b(g) .
$$

If no confusion arises, we shall denote a quadruple as above by a box as follows:

$$
h \prod_{y}^{x} g \text {. }
$$




\section{$\square(\mathscr{V}, \mathscr{H}) \rightrightarrows \mathscr{H}$}

The collection

$\begin{array}{lll}\downarrow & \downarrow \\ \mathscr{V} & \rightrightarrows & \mathscr{P}\end{array}$

forms a double groupoid in the way, called the $\rightrightarrows \mathscr{P}$

coarse double groupoid with sides in $\mathscr{H}$ and $\mathscr{V}$.

Definition 1.5 [Andruskiewitsch and Natale 2009]. A double groupoid $(\mathscr{B} ; \mathscr{V}, \mathscr{H} ; \mathscr{P})$ is slim if the morphism of (discrete) double groupoids $\Pi: \mathscr{B} \rightarrow \square(\mathscr{V}, \mathscr{H})$ given by

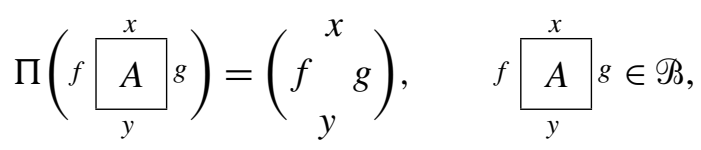

is injective. That is, any box in $\mathscr{B}$ is determined by its sides.

We now pass to the context of double Lie groupoids. Let $M, N$ and $P$ be smooth manifolds, let $f: M \rightarrow P$ and $g: N \rightarrow P$ be smooth maps, we remind that $f$ and $g$ are called transversal at $p \in \mathscr{P}$, if for every $m \in M$ and $n \in N$ such that $p=f(m)=g(n)$ the condition $\left(T_{m} f\right)\left(T_{m} M\right)+\left(T_{n} g\right)\left(T_{n} N\right)=T_{p} P$ holds. We said that $f$ and $g$ are transversal if they are transversal at every $p$ as above.

Remark 1.6. Let $\mathscr{V}$ and $\mathscr{H}$ be Lie groupoids over the same manifold $\mathscr{P}$. If the anchors maps $\chi_{\mathscr{V}}: \mathscr{V} \rightarrow \mathscr{P} \times \mathscr{P}$ and $\chi_{\mathscr{H}}: \mathscr{H} \rightarrow \mathscr{P} \times \mathscr{P}$ are transversal then $\square(\mathscr{V}, \mathscr{H})$ is a double Lie groupoid [Brown and Mackenzie 1992].

\section{Diagrams of groupoids}

The following notions were introduced in [Andruskiewitsch and Natale 2009], in the context of discrete double groupoids. Let $\mathscr{V}$ and $\mathscr{H}$ be groupoids over the same base $\mathscr{P}$. A diagram over $\mathscr{H}$ and $\mathscr{V}$ is a triple $(\mathscr{D}, j, i)$ where $\mathscr{D}$ is a groupoid over $\mathscr{P}$ and $i: \mathscr{H} \rightarrow \mathscr{D}, j: \mathscr{V} \rightarrow \mathscr{D}$ are morphisms of groupoids over $\mathscr{P}$. A diagram of groupoids $(\mathscr{D}, j, i)$ over $\mathscr{V}$ and $\mathscr{H}$ is called a $(\mathscr{V}, \mathscr{H})$-factorization of $\mathscr{D}$ if $\mathscr{D}=$ $j(\mathscr{V}) i(\mathscr{H})$.

To each diagram of groupoids $(\mathscr{D}, j, i)$ we associate a discrete double groupoid, denoted by $\square(\mathscr{D}, j, i)$ and defined as the double subgroupoid of $\square(\mathscr{V}, \mathscr{H})$ whose boxes are of the form

$$
A=h \square_{y}^{x} g \in \square(\mathscr{V}, \mathscr{H}),
$$

with $x, y \in \mathscr{H}, g, h \in \mathscr{V}$, such that $i(x) j(g)=j(h) i(y)$ in $\mathscr{D}$.

\section{Diagrams of Lie groupoids.}

Definition 2.1. Let $\mathscr{V}$ and $\mathscr{H}$ be Lie groupoids over $\mathscr{P}$. A diagram of Lie groupoids over $\mathscr{H}$ and $\mathscr{V}$ is a diagram of groupoids $(\mathscr{D}, j, i)$ such that $\mathscr{D}$ is a Lie groupoid and the maps $i$ and $j$ are smooth. If this diagram is a factorization in the sense above, then we say that it is a $(\mathscr{V}, \mathscr{H})$-factorization of the Lie groupoid $\mathscr{D}$. 
Let $(\mathscr{D}, j, i)$ be a diagram of Lie groupoids over $\mathscr{H}$ and $\mathscr{V}$. Our aim in this Subsection is to determine when $\square(\mathscr{D}, j, i)$ is a double Lie groupoid. For this, we shall consider two maps; the first one is the composition

$$
\mathscr{H}_{r} \times_{t} \mathscr{V} \stackrel{i \times j}{\longrightarrow} \mathscr{D}_{e} \times_{s} \mathscr{D} \stackrel{m}{\longrightarrow} \mathscr{D},
$$

that is,

$$
\Phi: \mathscr{H}_{r} \times_{t} \mathscr{V} \rightarrow \mathscr{D}, \quad(x, g) \mapsto i(x) j(g),
$$

where $s, e$ and $m$ are the source, target and composition maps of $\mathscr{D}$. The second one is

$$
\Psi: \mathscr{V}_{b} \times_{l} \mathscr{H} \rightarrow \mathscr{D}, \quad(f, y) \mapsto j(f) i(y) .
$$

Since $t, b, l$ and $r$ are surjective submersions, we have that the fiber products involved in the above maps, $\mathscr{V}_{b} \times_{l} \mathscr{H}$ and $\mathscr{H}_{r} \times_{t} \mathscr{V}$, are embedded submanifolds of $\mathscr{V} \times \mathscr{H}$ and $\mathscr{H} \times \mathscr{V}$ respectively, and since $i$ and $j$ are smooth then $\Phi$ and $\Psi$ also are. With the above maps

$$
\square(\mathscr{D}, j, i)=\left(\mathscr{V}_{b} \times_{l} \mathscr{H}\right) \Psi \times_{\Phi}\left(\mathscr{H}_{r} \times_{t} \mathscr{V}\right),
$$

and from general transversality theory [Lang 2002, Proposition 2.5], if $\Phi$ and $\Psi$ are transversal then $\square(\mathscr{D}, j, i)$ is an embedded submanifold of $\left(\mathscr{V}_{b} \times{ }_{l} \mathscr{H}\right) \times\left(\mathscr{H}_{r} \times{ }_{t} \mathscr{V}\right)$.

Lemma 2.2. Let $(\mathscr{D}, j, i)$ be a diagram of Lie groupoids. If $i$ and $j$ are transversal at the identities, then $\Psi$ and $\Phi$ defined above are submersions.

Proof. We take $((f, y),(x, g)) \in\left(\mathscr{V}_{b} \times{ }_{r} \mathscr{H}\right) \times\left(\mathscr{H}_{r} \times_{t} \mathscr{V}\right)$ such that $\Psi(f, y)=\Phi(x, g)$ i.e, $j(f) i(y)=i(x) j(g)$. Now, by [Lang 2002, Proposition 2.5], we have

$$
T_{(x, g)}\left(\mathscr{H}_{r} \times_{t} \mathscr{V}\right)=\left\{(Y, X) \in\left(T_{x} \mathscr{H}\right) \times\left(T_{g} \mathscr{V}\right) /\left(T_{x} r\right)(Y)=\left(T_{g} t\right)(X)\right\} .
$$

Let $W \in T_{(x, g)}\left(\mathscr{H}_{r} \times_{t} \mathscr{V}\right)$. We need to prove that there is $\left(X_{1}, Y_{1}\right) \in T_{(f, y)}\left(\mathscr{V}_{b} \times_{l} \mathscr{H}\right)$ such that $T_{(f, y)} \Psi\left(X_{1}, Y_{1}\right)=W$. Note that

$$
\begin{aligned}
T_{(f, y)} \Psi\left(X_{1}, Y_{1}\right) & =\left(T_{(j(f), i(y))} m\right)\left(T_{(f, y)} j \times i\right)\left(X_{1}, Y_{1}\right) \\
& =\left(T_{(j(f), i(y))} m\right)\left(\left(T_{f} j\right)\left(X_{1}\right),\left(T_{y} i\right)\left(Y_{1}\right)\right) .
\end{aligned}
$$

Now, we know that in $\mathscr{H} \rightrightarrows \mathscr{P}$ there is a local bisection $\tau: U \rightarrow \mathscr{H}$ with $r(y) \in U \subseteq \mathscr{P}$ open and $\tau(r(y))=y$ [Mackenzie 2005, Proposition 1.4.9]. Since $\tau$ is a bisection, it induces local left and right translations as in (1-1). Concretely, set $V=(l \circ \tau)(U)$ open in $\mathscr{P}, \mathscr{H}^{U}=l^{-1}(U)$ and $\mathscr{H}_{U}=r^{-1}(U)$ and define

$$
\begin{aligned}
& L_{\tau}: \mathscr{H}^{U} \rightarrow \mathscr{H}^{V}, \quad z \mapsto \tau(l(z)) z, \\
& R_{\tau}: \mathscr{H}_{V} \rightarrow \mathscr{H}_{U}, \quad z \mapsto z \tau\left((l \circ \tau)^{-1} r(z)\right) .
\end{aligned}
$$

Define the map $\tau_{\mathscr{D}}: U \rightarrow \mathscr{D}$ by $i \circ \tau$; since $i$ is a morphism of groupoids, $\tau_{\mathscr{D}}$ is a local bisection of $\mathscr{D}$. Also note that $\tau_{\mathscr{D}}(e(i(y)))=(i \circ \tau)(r(y))=i(y)$. 
Analogously, there is a local bisection $\sigma: U^{\prime} \rightarrow \mathscr{V}$ such that $\sigma_{\mathscr{V}}(b(f))=f$, with $U^{\prime} \subseteq \mathscr{D}$ open and $b(f) \in U^{\prime}$. Again this induces a local bisection $\sigma_{\mathscr{D}}: U^{\prime} \rightarrow \mathscr{D}$ such that $\sigma_{\mathscr{D}}(e(j(f)))=j(f)$. Let $\left(X_{1}, Y_{1}\right) \in T_{(f, y)}\left(\mathscr{V}_{b} \times_{l} \mathscr{H}\right)$, then by Xu's formula for product in the tangent groupoid [Mackenzie 2005, Theorem 1.4.14] we obtain:

$$
\begin{aligned}
T_{(f, y)} \Psi\left(X_{1}, Y_{1}\right) & =\left(T_{(j(f), i(y))} m\right)\left(\left(T_{f} j\right)\left(X_{1}\right),\left(T_{y} i\right)\left(Y_{1}\right)\right) \\
& =\left(T_{i(y)} L_{\sigma_{\mathscr{D}}}\right)\left(T_{y} i\right)\left(Y_{1}\right)+\left(T_{j(f)} R_{\tau_{\mathscr{D}}}\right)\left(T_{f} j\right)\left(X_{1}\right) \\
& -\left(T_{i(y)} L_{\sigma_{\mathscr{D}}}\right)\left(T_{\mathrm{id}_{\mathscr{D}} l(y)} R_{\tau_{\mathscr{D}}}\right)\left(T_{l(y)} \operatorname{id}_{\mathscr{D}}\right)(z),
\end{aligned}
$$

where we write $z=\left(T_{j(f)} e\right)\left(T_{f} j\right)\left(X_{1}\right)=\left(T_{i(y)} s\right)\left(T_{y} i\right)\left(Y_{1}\right)$.

Now $\left(T_{j(f) i(y)} L_{\sigma_{\mathscr{D}}}^{-1}\right)(W) \in T_{i(y)} \mathscr{D}$ because we have

$$
\begin{aligned}
L_{\sigma_{\mathscr{D}}}^{-1}(j(f) i(y)) & =\sigma_{\mathscr{D}}^{-1}(s(j(f) i(y))) j(f) i(y) \\
& =\sigma_{\mathscr{D}}\left(\left(s \circ \sigma_{\mathscr{D}}\right)^{-1}(s(j(f) i(y)))\right)^{-1} j(f) i(y) \\
& =\sigma_{\mathscr{D}}\left(\left(s \circ \sigma_{\mathscr{D}}\right)^{-1} s(j(f))\right)^{-1} j(f) i(y) \\
& =\sigma_{\mathscr{D}}(e(j(f)))^{-1} j(f) i(y) \\
& =j(f)^{-1} j(f) i(y)=i(y) .
\end{aligned}
$$

In an analogous way, we have $\left(T_{i(y)} R_{\tau_{\mathscr{D}}}^{-1}\right)\left(T_{j(f) i(y)} L_{\sigma_{\mathscr{D}}}^{-1}\right)(W) \in T_{\mathrm{Id}_{\mathscr{D}} l(y)} \mathscr{D}$ since

$$
\begin{aligned}
R_{\tau_{\mathscr{D}}}^{-1}(i(y)) & =i(y) \tau_{\mathscr{D}}^{-1}\left(\left(s \circ \tau_{\mathscr{D}}^{-1}\right)^{-1} e(i(y))\right) \\
& =i(y) \tau_{\mathscr{D}}\left(\left(s \circ \tau_{\mathscr{D}}\right)^{-1}\left(\left(s \circ \tau_{\mathscr{D}}^{-1}\right)^{-1} e(i(y))\right)\right)^{-1} \\
& =i(y) \tau_{\mathscr{D}}\left(\left(s \circ \tau_{\mathscr{D}}\right)^{-1}\left(\left(s \circ \tau_{\mathscr{D}}\right) e(i(y))\right)\right)^{-1} \\
& =i(y) \tau_{\mathscr{D}}(e(i(y)))^{-1}=i(y)^{-1} i(y)=\operatorname{Id}_{\mathscr{D}} s(i(y))=\operatorname{Id}_{\mathscr{D}}(l(y)) .
\end{aligned}
$$

Write $p=l(y)$, since $i$ and $j$ are transversal at $\operatorname{Id}_{\mathscr{D}}(p)$ then

$$
T_{\operatorname{Id}_{p}} \mathscr{D}=\left(T_{\operatorname{Id}_{p}} i\right)\left(T_{\operatorname{Id}_{p}} \mathscr{H}\right)+\left(T_{\operatorname{Id}_{p}} j\right)\left(T_{\operatorname{Id}_{p}} \mathscr{V}\right)
$$

and in consequence we can find $X \in T_{\mathrm{Id}_{p}} \mathscr{H}$ and $Y \in T_{\mathrm{Id}_{p}} \mathscr{V}$ such that

$$
\left(T_{i(y)} R_{\tau_{\mathscr{D}}}^{-1}\right)\left(T_{j(f) i(y)} L_{\sigma_{\mathscr{D}}}^{-1}\right)(W)=\left(T_{\mathrm{Id}_{p}} i\right)(X)+\left(T_{\operatorname{Id}_{p}} j\right)(Y) .
$$

Thus, if we take the vectors

$$
X^{\prime}=X+\left(T_{p} \operatorname{Id}_{\mathscr{H}}\right)\left(T_{\operatorname{Id}_{p}} t\right)(Y), \quad Y^{\prime}=Y+\left(T_{p} \operatorname{Id}_{\mathscr{V}}\right)\left(T_{\operatorname{Id}_{p}} l\right)(X),
$$

a direct calculation shows that

$(2-1) \quad\left(T_{i(y)} R_{\tau_{\mathscr{D}}}^{-1}\right)\left(T_{j(f) i(y)} L_{\sigma_{\mathscr{D}}}^{-1}\right)(W)=\left(T_{\mathrm{Id}_{p}} i\right)\left(X^{\prime}\right)+\left(T_{\operatorname{Id}_{p}} j\right)\left(Y^{\prime}\right)-\left(T_{p} \operatorname{Id}_{\mathscr{D}}\right)(Z)$ where $Z=\left(T_{\operatorname{Id}_{p}} b\right)(Y)+\left(T_{\operatorname{Id}_{p}} l\right)(X)$. 
Since $\left(L_{\sigma_{\mathscr{D}}} \circ R_{\tau_{\mathscr{D}}} \circ i\right)=\left(L_{\sigma_{\mathscr{D}}} \circ i \circ R_{\tau}\right)$ and $\left(L_{\sigma_{\mathscr{D}}} \circ R_{\tau_{\mathscr{D}}} \circ j\right)=\left(R_{\tau_{\mathscr{D}}} \circ j \circ L_{\sigma_{V}}\right)$, we may apply $\left(T_{i(y)} L_{\sigma_{D}}\right)\left(T_{\operatorname{Id}_{p}} R_{\tau_{\Im}}\right)$ to both sides of (2-1) and arrive at

$W=$

$$
\left(T_{i(y)} L_{\sigma_{\mathscr{D}}}\right)\left(T_{y} i\right)\left(Y_{1}\right)+\left(T_{j(f)} R_{\tau_{\mathscr{D}}}\right)\left(T_{f} j\right)\left(X_{1}\right)-\left(T_{i(y)} L_{\sigma_{\mathscr{D}}}\right)\left(T_{\mathrm{Id}_{p}} R_{\tau_{\mathscr{D}}}\right)\left(T_{p} \operatorname{Id}_{\mathscr{D}}\right)(Z),
$$

where $X_{1}=\left(T_{\operatorname{Id}_{p}} L_{\sigma_{V}}\right)\left(Y^{\prime}\right)$ and $Y_{1}=\left(T_{\operatorname{Id}_{p}} R_{\tau}\right)\left(X^{\prime}\right)$. It is clear that $\left(T_{f} b\right)\left(X_{1}\right)=$ $Z=\left(T_{y} l\right)\left(Y_{1}\right)$, thus $T_{(f, y)} \Psi\left(X_{1}, Y_{1}\right)=W$.

In the same way we prove that $\Phi$ is a submersion.

From the above result we obtain the following immediate consequence.

Theorem 2.3. Let $(\mathscr{D}, j, i)$ be a $(\mathscr{V}, \mathscr{H})$-factorization of the Lie groupoid $\mathscr{D}$. If $i$ and $j$ are transversal at the identities, then $\square(\mathscr{D}, j, i)$ is a slim double Lie groupoid.

Proof. By Lemma 2.2 we have that $\Phi$ and $\Psi$ are transversal, thus $\square(\mathscr{D}, j, i)$ is an embedded submanifold of $\left(\mathscr{V}_{b} \times_{l} \mathscr{H}\right) \times\left(\mathscr{H}_{r} \times_{t} \mathscr{V}\right)$. Since $\Phi$ and $\Psi$ are surjective submersions, both projections from $\square(\mathscr{D}, j, i)=\left(\mathscr{V}_{b} \times_{l} \mathscr{H}\right)_{\Psi} \times_{\Phi}\left(\mathscr{H}_{r} \times_{t} \mathscr{V}\right)$ to $\left(\mathscr{V}_{b} \times_{l} \mathscr{H}\right)$ and $\left.\mathscr{H}_{r} \times_{t} \mathscr{V}\right)$ are surjective submersions and the same is true for the projections from the fiber products $\mathscr{V}_{b} \times_{l} \mathscr{H}$ and $\mathscr{H}_{r} \times_{t} \mathscr{V}$ to $\mathscr{V}$ and $\mathscr{H}$; then the top, bottom, left and right maps from $\square(\mathscr{D}, j, i)$ are surjective submersions and the same for the top-right corner map. It is clear that the compositions, the identities maps and the inversions maps are smooth.

The diagonal groupoid. In this subsection, all groupoids are discrete. We review from [Andruskiewitsch and Natale 2009] the construction of the diagonal groupoid. Let $\mathscr{B}$ be a double groupoid that satisfies the filling condition and let $\mathscr{V} \circledast \mathscr{H}$ be the free product (over $\mathscr{P}$ ) of the vertical and horizontal groupoids; see the same reference and [Higgins 2005]. We set

$$
[A]:=x g y^{-1} h^{-1} \in \mathscr{V} \circledast \mathscr{H} \quad \text { if } \frac{h_{y}}{A} g \in \mathscr{B} .
$$

We write $J_{\circledast}(\mathscr{B})$ for the subgroupoid of $\mathscr{V} \circledast \mathscr{H}$ generated by $\{[A] \mid A \in \mathscr{B}\}$. As $s_{\circledast}([A])=e_{\circledast}([A])=t l(A)$ we have that the groupoid $J_{\circledast}(\mathscr{B}) \rightrightarrows \mathscr{P}$ is in fact a group bundle. We know that the group bundle $J_{\circledast}(\mathscr{B})$ is a normal subgroupoid of $\mathscr{V} \circledast \mathscr{H}$ [Andruskiewitsch and Natale 2009, Lemma 3.5].

Assume that $(\mathscr{B} ; \mathscr{V}, \mathscr{H} ; \mathscr{P})$ is slim; then the associated diagonal groupoid is the quotient $\mathscr{D}(\mathscr{B})=\mathscr{V} \circledast \mathscr{H} / J_{\circledast}(\mathscr{B})$. If we compose the natural inclusions of $\mathscr{V}$ and $\mathscr{H}$ in $\mathscr{V} \circledast \mathscr{H}$ with the projections to $\mathscr{D}(\mathscr{B})$ we get two groupoid morphisms

$$
i: \mathscr{H} \rightarrow \mathscr{D}(\mathscr{B}) \text { and } j: \mathscr{V} \rightarrow \mathscr{D}(\mathscr{B}) \text {. }
$$


Thus we have a $(\mathscr{V}, \mathscr{H})$-factorization $(\mathscr{D}(\mathscr{B}), i, j)$. In this subsection our aim is to give another presentation of the diagonal groupoid as a quotient of $\mathscr{V}_{b} \times_{l} \mathscr{H}$.

Proposition 2.4. Let $(\mathscr{B} ; \mathcal{V}, \mathscr{H} ; \mathscr{P})$ be a slim double groupoid that satisfies the filling condition. We define on $\mathcal{V}_{b} \times_{l}$ H the following relation $\sim_{\mathscr{B}}$ :

$$
\left(v_{1}, h_{1}\right) \sim_{\mathscr{B}}\left(v_{2}, h_{2}\right) \Longleftrightarrow r\left(h_{1}\right)=r\left(h_{2}\right), t\left(v_{1}\right)=t\left(v_{2}\right) \text { and } v_{1} h_{1} h_{2}^{-1} v_{2}^{-1} \in J_{\circledast}(\mathscr{B}) \text {. }
$$

Then $\sim_{\mathscr{B}}$ is an equivalence relation and the map

$$
\phi: \mathscr{V}_{b} \times_{l} \mathscr{H} / \sim_{\mathscr{B}} \rightarrow \mathscr{D}(\mathscr{B}), \quad[v, h] \mapsto j(v) i(h)
$$

is a well defined isomorphism of quivers over $\mathscr{P}$.

Proof. Clearly $\sim_{\mathscr{B}}$ is an equivalence relation; we write $\mathscr{G}:=\left(\mathscr{V}_{b} \times_{l} \mathscr{H}\right) / \sim_{\mathscr{B}}$ and denote by $[f, x]$ the equivalence class of $(f, x) \in \mathscr{V}_{b} \times_{l} \mathscr{H}$. Let $\left(f_{1}, x_{1}\right),\left(f_{2}, x_{2}\right)$ be elements of $\mathscr{V}_{b} \times_{l} \mathscr{H}$ such that $\left[f_{1}, x_{1}\right]=\left[f_{2}, x_{2}\right]$, then we have $f_{1} x_{1} x_{2}^{-1} f_{2}^{-1} \in$ $J_{\circledast}(\mathscr{B})$ so that $\overline{f_{1}} \overline{x_{1}}=\overline{f_{2}} \overline{x_{2}}$ in $\mathscr{D}(\mathscr{B})$ (where $\bar{w}$ denotes the image of $w$ under $i$ if $w$ belongs to $\mathscr{H}$, or under $j$ if $w$ belongs to $\mathscr{V})$. This proves that $\phi$ is well defined.

Suppose that $g, g^{\prime} \in \mathscr{V}$ and $x, x^{\prime} \in \mathscr{H}$ is any collection satisfying $\bar{g} \bar{x}=\overline{g^{\prime}} \overline{x^{\prime}}$. Then $g^{\prime} x^{\prime} x^{-1} g^{-1} \in J_{\circledast}(\mathscr{B})$, hence $\left[g^{\prime}, x^{\prime}\right]=[g, x]$. Therefore $\phi$ is injective.

To prove that $\phi$ is surjective, let $d \in \mathscr{D}(\mathscr{B})$. Then $d=\overline{d_{1}} \overline{d_{2}} \cdots \overline{d_{n}}$ with $d_{i}$ an element of $\mathscr{V}$ or $\mathscr{H}$. Let $d_{i} \in \mathscr{H}, d_{i+1} \in \mathscr{V}$ with $r\left(d_{i}\right)=t\left(d_{i+1}\right)$. Since $\mathscr{B}$ satisfies the filling condition, the corner

$$
\frac{d_{i}}{{ }^{\prime}} d_{i+1}
$$

can be completed to a box in $\mathscr{B}$; that is, there exists $B \in \mathscr{B}$ such that

$$
B=f \prod_{y}^{d_{i}} d_{i+1} .
$$

Thus, $d_{i} d_{i+1} y^{-1} f^{-1} \in J_{\circledast}(\mathscr{B})$ and $\overline{d_{i}} \overline{d_{i+1}}=\bar{f} \bar{y}$. So, we can commute the $d_{i}$ 's in $d=\overline{d_{1}} \overline{d_{2}} \cdots \overline{d_{n}}$ in such a way that we can obtain $d=\bar{g} \bar{x}$ with $g \in \mathscr{V}, x \in \mathscr{H}$ and $b(g)=l(x)$. This proves that $\phi$ is surjective.

Remark 2.5. The map $\phi$ from the previous lemma induces a structure of groupoid on $\mathscr{G}=\mathscr{V}_{b} \times_{l} \mathscr{H} / \sim_{\mathscr{B}}$ with the following structural maps:

- The source and the target projections are

$$
s: \mathscr{G} \rightarrow \mathscr{P}, \quad[v, h] \mapsto t(v) ; \quad e: \mathscr{G} \rightarrow \mathscr{P}, \quad[v, h] \mapsto r(h) .
$$

- The identity map is id: $\mathscr{P} \rightarrow \mathscr{G}, \quad p \mapsto \operatorname{id}_{p}=\left[\operatorname{id}_{p}, \mathrm{id}_{p}\right]$. 
- Multiplication is given by $\left[v_{1}, h_{1}\right]\left[v_{2}, h_{2}\right]=\left[v_{1} f, z h_{2}\right]$, where $f \prod_{z} v_{2} \in \mathscr{B}$,
when it is defined;

- The inverse map is given by $[v, h]^{-1}=\left[f^{-1}, z^{-1}\right]$, for every $[v, h] \in \mathscr{G}$ and where $v \underset{h}{z} f \in \mathscr{B}$.

Now we recall the following lemma which will be very useful for our purposes.

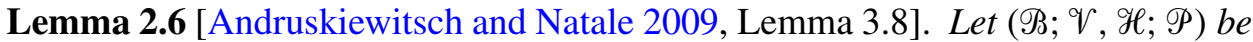
a slim double groupoid that satisfies the filling condition. Let $f \in \mathscr{V}$ and $x \in \mathcal{H}$ such that:

- $l(x)=b(f)$ and $t(f)=r(x)$,

- $f x \in J_{\circledast}(\mathscr{B}) \subset \mathscr{V} \circledast \mathscr{H}$.

Then there exists $E \in \mathbf{E}(\mathscr{B})$ such that $E=f \underset{x}{\bar{\square}}$.

We give an alternative description of $\sim_{\mathscr{B}}$ using this lemma. Indeed, $\left(f_{1}, x_{1}\right) \sim_{\mathscr{B}}$ $\left(f_{2}, x_{2}\right)$ if and only if $f_{1} x_{1} x_{2}^{-1} f_{2}^{-1} \in J_{\circledast}(\mathscr{B})$. Since $J_{\circledast}(\mathscr{B})$ is a normal subgroupoid, we have that $\left(f_{1}, x_{1}\right) \sim_{\mathscr{B}}\left(f_{2}, x_{2}\right)$ if and only if $f_{2}^{-1} f_{1} x_{1} x_{2}^{-1} \in J_{\circledast}(\mathscr{B})$. Hence

$$
\left(f_{1}, x_{1}\right) \sim_{\mathscr{B}}\left(f_{2}, x_{2}\right) \quad \Longleftrightarrow \quad \text { there exists } \quad E=\frac{f_{2}^{-1} f_{1} \square}{x_{1} x_{2}^{-1}} \in \mathscr{B} .
$$

Thus, the graph of the relation $\sim_{\mathscr{B}}$ is

$R=\left\{\left(f_{1}, x_{1}, f_{2}, x_{2}\right) \in\left(\mathscr{V}_{b} \times_{l} \mathscr{H}\right)_{\eta} \times_{\eta}\left(\mathscr{V}_{b} \times_{l} \mathscr{H}\right) \mid \exists E \in \mathbf{E}(\mathscr{B}), E={ }^{f_{2}^{-1} f_{1}}{\left.\underset{x_{1} x_{2}^{-1}}{\square}\right\}}\right.$ where

$$
\eta: \mathscr{V}_{b} \times_{l} \mathscr{H} \rightarrow \mathscr{P} \times \mathscr{P}, \quad(f, x) \mapsto(t(f), r(x)) ;
$$

from this we infer that the relation $\sim_{\mathscr{B}}$ is determined by the core groupoid of $\mathscr{B}$.

Lemma 2.7. Let $(\mathscr{B} ; \mathscr{V}, \mathscr{H} ; \mathscr{P})$ be a slim double groupoid satisfying the filling condition. If $\left(f_{1}, x_{1}\right),\left(f_{2}, x_{2}\right) \in \mathscr{V}_{b} \times_{l} \mathscr{H}$, then $\left(f_{1}, x_{1}\right) \sim_{\mathscr{B}}\left(f_{2}, x_{2}\right)$ if and only if there exist $A, B \in \mathscr{B}$ such that

$$
A=f_{1} \square_{x_{1}}^{x} g, \text { and } B=f_{2} \underset{x_{2}}{\square_{x}} g .
$$

Proof. In fact, if $A=f_{1} \square_{x_{1}}^{x} g$ and $B=f_{2} \prod_{x_{2}}^{x} g$ belong to $\mathscr{B}$, then $x g x_{1}^{-1} f_{1}^{-1} \in$ $J_{\circledast}(\mathscr{B})$ and $x g x_{2}^{-1} f_{2}^{-1} \in J_{\circledast}(\mathscr{B})$; taking the inverse of the first one and composing with the second one we arrive at $f_{1} x_{1} x_{2}^{-1} f_{2}^{-1} \in J_{\circledast}(\mathscr{B})$, that is, $\left(f_{1}, x_{1}\right) \sim_{\mathscr{B}}\left(f_{2}, x_{2}\right)$. 
Conversely, if $\left(f_{1}, x_{1}\right) \sim_{\mathscr{B}}\left(f_{2}, x_{2}\right)$ then by (2-3) there is $E \in \mathbf{E}(\mathscr{B})$ such that

$$
E=f_{2}^{-1} f_{1} \underset{x_{1} x_{2}^{-1}}{\square} \text {. }
$$

The filling condition guarantees that given $x_{2} \in \mathscr{H}$ and $f_{2} \in \mathscr{V}$ with $l\left(x_{2}\right)=b\left(f_{2}\right)$, there is a box $B^{\prime} \in \mathscr{B}$ with $t\left(B^{\prime}\right)=x_{2}$ and $l\left(B^{\prime}\right)=f_{2}^{-1}$. Let

$$
B^{\prime}=f_{2}^{-1} \prod_{x}^{x_{2}} g^{-1} \quad \text { and } \quad A^{\prime}:=\left\{\begin{array}{cc}
E^{v} & \mathbf{i d}\left(x_{2}\right) \\
\mathbf{i d}\left(f_{2}\right) & B^{\prime}
\end{array}\right\}=f_{1}^{-1} \underset{x}{\square_{1}} g^{-1} .
$$

Let $A, B$ be the vertical inverses of $A^{\prime}$ and $B^{\prime}$. Thus $A=f_{1} \underset{x_{1}}{\square} g$ and $B=f_{2} \frac{x}{x_{2}} g$
are both in $\mathscr{B}$ and we get the result. $\quad \square$

\section{Slim double Lie groupoids}

\section{Actions of Lie groupoids.}

Definition 3.1. A left action of a groupoid $\mathscr{G} \rightrightarrows \mathscr{P}$ along a map $\varepsilon: N \rightarrow \mathscr{P}$ is given by a map $\mathscr{G}_{e} \times{ }_{\varepsilon} N \rightarrow N$, denoted by $(g, n) \mapsto g n$, such that:

$$
\varepsilon(h y)=s(h), \quad \operatorname{id}(\varepsilon(y)) y=y, \quad(g h) y=g(h y),
$$

for all $g, h \in \mathscr{G}$ and $y \in N$ such that $e(g)=s(h)$ and $e(h)=\varepsilon(y)$. We shall simply say that the groupoid $\varphi$ acts on $N$.

The transformation or action groupoid $\varphi \ltimes N \rightrightarrows N$, associated with such an action, is a groupoid with set of arrows $\mathscr{G}_{e} \times{ }_{\varepsilon} N$. The source and target maps are

$$
s^{\prime}, e^{\prime}: \mathscr{G} \ltimes N \rightarrow N, \quad \text { given by } \quad s^{\prime}(g, n)=g n \quad \text { and } \quad e^{\prime}(g, n)=n \text {, }
$$

respectively, and composition $(g, n)(h, m)=(g h, m)$.

Let $\varphi \rightrightarrows \mathscr{P}$ be a Lie groupoid, $N$ a smooth manifold and $\varepsilon: N \rightarrow \mathscr{P}$ a smooth map. A left action is smooth provided that the map $\mathscr{G}_{e} \times{ }_{\varepsilon} N \rightarrow N$ is smooth. The resulting action groupoid is again a Lie groupoid.

The canonical left action of a groupoid $\mathscr{G} \rightrightarrows \mathscr{P}$ along id $: \mathscr{P} \rightarrow \mathscr{P}$ is given by $g p=s(g)$, where $g \in \mathscr{G}, p \in \mathscr{P}$ and $e(g)=p$. Recall that the orbit space $\mathscr{P} / \mathscr{G}$ is the quotient space of $\mathscr{P}$ where two points of $\mathscr{P}$ are identified if there exists an arrow between them. Thus, if we have $\mathscr{G} \rightrightarrows \mathscr{P}$ an action along a map $\varepsilon: N \rightarrow \mathscr{P}$, the orbit space $N / \mathscr{G}$ is equal to $N / \mathscr{G} \ltimes N$, the orbit space of the action groupoid. Also, the stabilizer of $n \in N$ is defined in the usual way; it coincides with the isotropy group of $n$ with respect to the action groupoid.

We recall that a continuous map $f: X \rightarrow Y$ between two topological spaces $X$ and $Y$, is said to be proper if the inverse image of a compact subset of $Y$ is compact. 
Definition 3.2. A Lie groupoid $\mathscr{G} \rightrightarrows \mathscr{P}$ is proper if the anchor map is proper. An action of a Lie groupoid $\mathscr{G}$ on a smooth manifold $N$ is proper if the action groupoid $\mathscr{G} \ltimes N$ is proper.

The following proposition is useful to decide when an action is proper.

Proposition 3.3 [Tu 2004, 2.14]. Let $\mathscr{G} \rightrightarrows \mathscr{P}$ be a Lie groupoid and $N$ a smooth manifold endowed with a left action of $\mathscr{G}$. Then $\mathscr{G}$ acts properly on $N$ if and only if the anchor map $\left(s^{\prime}, e^{\prime}\right): \mathscr{G} \ltimes N \rightarrow N \times N$ is closed and $\forall n \in N$ the stabilizer of $n$ is compact.

A groupoid is free when all the isotropy groups are trivial; thus, an action groupoid is free when all stabilizers are trivial.

Proposition 3.4 [Dragulete 2007, Theorem 3.3.1]. If $\mathscr{G} \rightrightarrows \mathscr{P}$ is a free and proper groupoid, then the orbit space $\mathscr{P} / \mathscr{G}$ is a smooth manifold and the orbit projection $\mathscr{P} \rightarrow \mathscr{P} / \mathscr{G}$ is a submersion.

In particular, if $\varphi$ is a Lie groupoid with a free and proper smooth action on $N$, then the orbit space $N / \mathscr{G}$ is a smooth manifold and the orbit projection $N \rightarrow N / \mathscr{G}$ is a submersion.

\section{The core action.}

Proposition 3.5 [Andruskiewitsch and Natale 2009, Proposition 1.1]. Suppose $(\mathscr{B} ; \mathscr{V}, \mathscr{H} ; \mathscr{P})$ is a slim double groupoid. Define $\gamma: \mathscr{B} \rightarrow \mathscr{P}, \gamma(A)=l b(A)$, the bottom-left vertex of $A$.

(a) There is an action of the core groupoid $\mathbf{E}(\mathscr{B})$ on $\gamma: \mathscr{B} \rightarrow \mathscr{P}$ given by

$$
E \rightarrow A:=\left\{\begin{array}{cc}
\operatorname{id} l(A) & A \\
E & \text { id } b(A)
\end{array}\right\}, \quad A \in \mathscr{B}, E \in \mathbf{E} .
$$

(b) Let $B \in \mathscr{B}$. Then the stabilizer $\mathbf{E}(\mathscr{B})^{B}$ is trivial and the orbit of $B$ is $\mathscr{O}_{B}=$ $\{A \in \mathscr{B}: t(A)=t(B), r(A)=r(B)\}$.

We introduce next the core action of the core groupoid on the fibred product $\mathscr{V}_{b} \times_{l} \mathscr{H}$, that allows us to present the diagonal groupoid as a quotient of $\mathscr{V}{ }_{b} \times_{l} \mathscr{H}$.

Lemma 3.6. Let $(\mathscr{B} ; \mathscr{V}, \mathscr{H} ; \mathscr{P})$ be a slim double groupoid and let $\eta: \mathscr{V}_{b} \times_{l} \mathscr{H} \rightarrow \mathscr{P}$ be given by $\eta(f, x)=b(f)=l(x)$. Then $\mathbf{E}(\mathscr{B})$ acts on $\eta$ by

$$
E \triangleright(f, x)=(f l(E), b(E) x), \quad \text { when } \eta(f, x)=e_{\mathbf{E}(\mathscr{B})}(E) .
$$

The orbit space $\mathscr{V}_{b} \times{ }_{l} \mathscr{H} / \mathbf{E}(\mathscr{B})$ coincides with $\mathscr{D}(\mathscr{B})$.

The map (3-2) is the core action of $\mathbf{E}(\mathscr{B})$ on $\mathscr{V}_{b} \times_{l} \mathscr{H}$. 
Proof. Since $b(f l(E))=b l(E)=s_{\mathbf{E}(\mathscr{B})}(E)$ and $l(b(E) x)=l b(E)=s_{\mathbf{E}(\mathscr{B})}(E)$, the map $\triangleright: \mathbf{E}(\mathscr{B})_{e_{\mathbf{E}(\mathscr{B})}} \times_{\eta}\left(\mathscr{V}_{b} \times_{l} \mathscr{H}\right) \rightarrow \mathscr{V}_{b} \times_{l} \mathscr{H}$ is well defined and $\triangleright$ is an action:

$$
\begin{aligned}
(E \circ F) \triangleright(f, x) & =(f l(E \circ F), b(E \circ F) x)=(f l(F) l(E), b(E) b(F) x) \\
& =E \triangleright(f l(F), t(F) x)=E \triangleright(F \triangleright(f, x)) .
\end{aligned}
$$

Also, $\eta(E \triangleright(f, x))=\eta(f l(E), b(E) x)=l(b(E) x)=l b(E)=s_{\mathbf{E}(\mathscr{B})}(E)$.

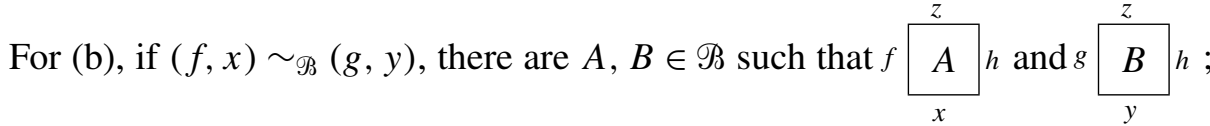
see Lemma 2.7. Then, by Proposition 3.5, there is a box $E \in \mathbf{E}(\mathscr{B})$ such that $A=E \rightarrow B$, in consequence, $x=b(E) y$ and $f=g l(E)$.

Conversely, if $A, B \in \mathscr{B}$ and $E \in \mathbf{E}(\mathscr{B})$ satisfy $b(A)=b(E) b(B)$ and $l(A)=$ $l(B) l(E)$, then the boxes $E \rightarrow B$ and $B$ have the same top and right sides. By Lemma 2.7, we have

$$
(l(E \rightarrow B), b(E \rightarrow B)) \sim_{\mathscr{B}}(l(B), b(B)),
$$

that is $(l(A), b(A)) \sim_{\mathscr{B}}(l(B), b(B))$. From this we conclude that given $(f, x)$ and $(g, y)$ in $\mathscr{V}_{b} \times_{l} \mathscr{H}$, we have $(f, x) \sim_{\mathscr{B}}(g, y)$ if and only if there exists $E \in \mathbf{E}(\mathscr{B})$ with $f=g l(E)$ and $x=b(E) y$. Thus the orbit space coincides with the diagonal groupoid.

Let $\pi: \mathscr{V}_{b} \times_{l} \mathscr{H} \rightarrow \mathscr{V}_{b} \times_{l} \mathscr{H} / \mathbf{E}(\mathscr{B})$ be the projection determined by (3-2). Let

$$
\begin{aligned}
& \tilde{i}: \mathscr{H} \rightarrow \mathscr{V}_{b} \times_{l} \mathscr{H}, \quad x \mapsto(\operatorname{id}(l(x)), x) \\
& \tilde{j}: \mathscr{V} \rightarrow \mathscr{V}_{b} \times_{l} \mathscr{H}, \quad f \mapsto(f, \operatorname{id}(b(f))),
\end{aligned}
$$

be the canonical inclusions of $\mathscr{V}$ and $\mathscr{H}$, and consider the compositions $i=\pi \circ \tilde{i}$ and $j=\pi \circ \tilde{j}$.

\section{The main theorem.}

Theorem 3.7. Let $\mathscr{V}$ and $\mathscr{H}$ be Lie groupoids. The assignments $\mathscr{B} \mapsto \mathscr{D}(\mathscr{B})$ and $(\mathscr{D}, j, i) \mapsto \square(\mathscr{D}, j, i)$ determine mutual category equivalences between

(a) the category of slim double Lie groupoids $(\mathscr{B} ; \mathscr{V}, \mathscr{H} ; \mathscr{P})$ with proper core action, and

(b) the category of $(\mathscr{V}, \mathscr{H})$-factorizations of Lie groupoids $(\mathscr{D}, j, i)$ such that the maps $i$ and $j$ are transversal at the identities.

Proof. The equivalence of categories at the discrete level was obtained in [Andruskiewitsch and Natale 2009]. We see that this equivalence behaves well in the differentiable context. We start with the direction from (a) to (b). 
Step 1. Let $(\mathscr{B} ; \mathscr{V}, \mathscr{H} ; \mathscr{P})$ be a slim double Lie groupoid. If the core action is proper, then $\mathscr{D}(\mathscr{B})$ is a Lie groupoid over $\mathscr{P}$. Furthermore, the maps $i$ and $j$ defined above are transversal at the identities.

Proof of the step. Since $\mathscr{B}$ is slim, the action (3-2) is free. Hence, the orbit space $\mathscr{V}_{b} \times_{l} \mathscr{H} / \mathbf{E}(\mathscr{B})$ has a unique manifold structure such that the canonical projection $\pi: \mathscr{V}_{b} \times_{l} \mathscr{H} \rightarrow \mathscr{V}_{b} \times_{l} \mathscr{H} / \mathbf{E}(\mathscr{B})$ is a surjective submersion, because the action is proper, see Proposition 3.4. We deduce using the identification in Lemma 3.6 that $\mathscr{D}(\mathscr{B})$ is a Lie groupoid over $\mathscr{P}$. In fact, the structure maps are described in Remark 2.5; using local sections of $\pi$, it is clear that the source and target maps are surjective submersions and that the other structural maps are smooth.

Let us now proceed with transversality. Let $p \in \mathscr{P}$ and we take a tangent vector

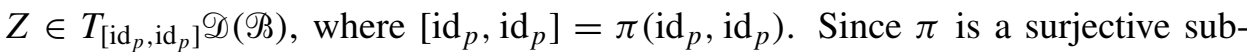
mersion, there is $(U, W) \in T_{\left(\mathrm{id}_{p}, \mathrm{id}_{p}\right)}\left(\mathcal{V}_{b} \times_{l} \mathscr{H}\right)$ such that $T_{\left(\mathrm{id}_{p}, \mathrm{id}_{p}\right)} \pi(U, W)=Z$. Choose

$$
Y=U \in T_{\operatorname{id}_{p}} \mathscr{V}, \quad X=W-\left(T_{p} \operatorname{id}_{\mathscr{H}}\right)\left(T_{\mathrm{id}_{p}} b\right)(U) \in T_{\operatorname{id}_{p}} \mathscr{H} .
$$

It is clear that

$$
\left(T_{\mathrm{id}_{p}} \tilde{j}\right)(Y)=\left(U,\left(T_{p} \operatorname{id}_{\mathscr{H}}\right)\left(T_{\operatorname{id}_{p}} b(U)\right)\right) \text { and }\left(T_{\operatorname{id}_{p}} \tilde{i}\right)(X)=\left(\left(T_{p} \operatorname{id}_{V}\right)\left(T_{\operatorname{id}_{p}} l\right)(X), X\right) .
$$

We compute

$$
\begin{aligned}
\left(T_{p} \mathrm{id}_{\mathscr{V}}\right)\left(T_{\mathrm{id}_{p}} l\right)(X) & =\left(T_{p} \mathrm{id}_{\mathscr{V}}\right)\left(T_{\mathrm{id}_{p}} l\right)(W)-\left(T_{p} \mathrm{id}_{\mathscr{V}}\right)\left(T_{\mathrm{id}_{p}} l\right)\left(T_{p} \mathrm{id}_{\mathscr{H}}\right)\left(T_{\mathrm{id}_{p}} b\right)(U) \\
& =\left(T_{p} \mathrm{id}_{\mathscr{V}}\right)\left(T_{\mathrm{id}_{p}} l\right)(W)-\left(T_{p} \mathrm{id}_{\mathscr{V}}\right)\left(T_{\mathrm{id}_{p}} b\right)(U)=0
\end{aligned}
$$

then $\left(T_{\operatorname{id}_{p}} \tilde{i}\right)(X)=(0, X)$. In consequence we have

$$
\begin{aligned}
\left(T_{\operatorname{id}_{p}} \tilde{j}\right)(Y)+\left(T_{\operatorname{id}_{p}} \tilde{i}\right)(X) & =\left(U,\left(T_{p} \operatorname{id}_{\mathscr{H}}\right)\left(T_{\operatorname{id}_{p}} b\right)(U)\right)+(0, X) \\
& =\left(U,\left(T_{p} \operatorname{id}_{\mathscr{H}}\right)\left(T_{\operatorname{id}_{p}} b\right)(U)+W-\left(T_{p} \operatorname{id}_{\mathscr{H}}\right)\left(T_{\operatorname{id}_{p}} b\right)(U)\right) \\
& =(U, W) .
\end{aligned}
$$

Then if we apply $T_{\left(\mathrm{id}_{p}, \mathrm{id}_{p}\right)} \pi$ to both sides of the above equation we arrive to

$$
\left(T_{\mathrm{id}_{p}} j\right)(Y)+\left(T_{\mathrm{id}_{p}} i\right)(X)=Z,
$$

that is, the maps $i$ and $j$ are transversal at the identities.

Step 2. Let $(\mathscr{D}, j, i)$ be a $(\mathscr{V}, \mathscr{H})$-factorization of Lie groupoids such that the maps $i$ and $j$ are transversal at the identities. Then the associated double groupoid $\square(\mathscr{D}, j, i)$ is a slim double Lie groupoid and its core action is proper.

Proof of the step. By Theorem 2.3, $\square(\mathscr{D}, j, i)$ is a slim double Lie groupoid. By [Brown and Mackenzie 1992], its core groupoid is a Lie groupoid with underlying 
manifold

$$
\mathscr{V}^{o p}{ }_{j} \times_{i} \mathscr{H}=\left\{(h, y) \mid j\left(h^{-1}\right)=i(y)\right\} .
$$

Up to this description, the core action on $\mathscr{V}_{b} \times_{l} \mathscr{H}$ is given by

$$
(h, y) \triangleright(f, x)=(f h, y x) \quad \text { when } \quad \eta(f, x)=t(h)=r(y) .
$$

It remains to prove that the core action (3-3) is proper. But the action (3-3) is free, that is the stabilizers are trivial. Thus, in order to prove that it is proper, we only need to check that the anchor map of the corresponding action groupoid

$$
(s, t):\left(\mathscr{V}^{o p}{ }_{j} \times_{i} \mathscr{H}\right) \ltimes\left(\mathscr{V}_{b} \times_{l} \mathscr{H}\right) \rightarrow\left(\mathscr{V}_{b} \times_{l} \mathscr{H}\right) \times\left(\mathscr{V}_{b} \times_{l} \mathscr{H}\right)
$$

is closed; see Proposition 3.3. Let $A \subseteq\left(\mathscr{V}_{j} \times_{i} \mathscr{H}\right) \ltimes\left(\mathscr{V}_{b} \times_{l} \mathscr{H}\right)$ be a closed set and consider a sequence $\left\{\left(f_{n}, x_{n}, g_{n}, y_{n}\right)\right\}_{n \in \mathbb{N}}$ in $A$ such that

$$
\left\{(s, t)\left(f_{n}, x_{n}, g_{n}, y_{n}\right)\right\}_{n \in \mathbb{N}}=\left\{\left(g_{n} f_{n}, x_{n} y_{n}, g_{n}, y_{n}\right)\right\}_{n \in \mathbb{N}}
$$

converges to $(a, b, g, y) \in\left(\mathscr{V}_{b} \times_{l} \mathscr{H}\right) \times\left(\mathscr{V}_{b} \times_{l} \mathscr{H}\right)$. We need to see that $(a, b, g, y) \in$ $(s, t)(A)$.

Clearly we have $g_{n} \rightarrow g, y_{n} \rightarrow y, g_{n} f_{n} \rightarrow a$, and $x_{n} y_{n} \rightarrow b$ as $n \rightarrow \infty$. Hence $\left(f_{n}, x_{n}, g_{n}, y_{n}\right) \rightarrow\left(g^{-1} a, b y^{-1}, g, y\right)$. Since $A$ is a closed set, we conclude that $\left(\left(g^{-1} a, b y^{-1}, g, y\right) \in A\right.$. But $(a, b, g, y)=(s, t)\left(g^{-1} a, b y^{-1}, g, y\right) \in(s, t)(A)$, hence $(s, t)(A)$ is closed. This finishes the proof of the step and of the theorem.

Example 3.8. Let $G$ be a simply connected Poisson-Lie group, $\mathfrak{g}$ its Lie algebra. It is well known that $\mathfrak{g}$ is a Lie bialgebra; let $\mathfrak{g}^{*}$ be the dual Lie algebra and let $\mathfrak{d}$ be the corresponding Drinfeld double. Let $G^{*}$ and $D$ be simply connected Lie groups with Lie algebras $\mathfrak{g}^{*}$ and $\mathfrak{d}$, respectively. Then the maps $G \rightarrow D$ taking $u$ to $\bar{u}$ and $G^{*} \rightarrow D$ taking $g \mapsto \bar{g}$ give rise to a symplectic double groupoid [Lu and Weinstein 1989, Theorem 3]:

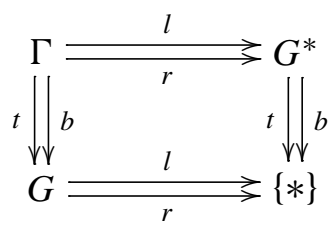

where $\Gamma=\left\{(g, u, v, h) \mid g, h \in G^{*}, u, v \in G\right.$, and $\left.\bar{g} \bar{u}=\bar{v} \bar{h}\right\}$.

It is clear that $\mathbf{E}(\Gamma)=\left\{(g, u, e, e) \mid g \in G^{*}, u \in G\right.$, and $\left.\bar{g} \bar{u}=e\right\}$, that the core action over $G \times G^{*}$ is given by $(g, v, e, e)(a, b)=(a g, v b)$, and that the anchor of the respective action groupoid is

$\mathfrak{X}: \mathbf{E}(\Gamma) \times\left(G \times G^{*}\right) \rightarrow\left(G \times G^{*}\right) \times\left(G \times G^{*}\right), \quad((g, v, e, e),(a, b))=(a g, v b)$. 
Arguing as in the in the proof of Step 2 of Theorem 3.7, we see that the core action is proper. Observe that $\left(\Gamma, G, G^{*}, *\right)$ does not satisfy, in general, the filling condition. If $\bar{G} \overline{G^{*}}=\overline{G^{*}} \bar{G}$, then $\Gamma$ is a slim double Lie groupoid.

\section{References}

[Andruskiewitsch and Natale 2005] N. Andruskiewitsch and S. Natale, "Double categories and quantum groupoids", Publ. Mat. Urug. 10 (2005), 11-51. MR 2006b:16058 Zbl 1092.16021 arXiv math.QA/0308228

[Andruskiewitsch and Natale 2006] N. Andruskiewitsch and S. Natale, "Tensor categories attached to double groupoids”, Adv. Math. 200:2 (2006), 539-583. MR 2007f:18008 Zbl 1099.16016

[Andruskiewitsch and Natale 2009] N. Andruskiewitsch and S. Natale, "The structure of double groupoids", J. Pure Appl. Algebra 213:6 (2009), 1031-1045. MR 2010e:20083 Zbl 1222.18004

[Brown 2004] R. Brown, "Crossed complexes and homotopy groupoids as non commutative tools for higher dimensional local-to-global problems", pp. 101-130 in Galois theory, Hopf algebras, and semiabelian categories (Toronto, ON, 2002), edited by G. Janelidze et al., Fields Institute Communications 43, American Mathematical Society, Providence, RI, 2004. MR 2005f:18001 Zbl 1067.18004

[Brown and Janelidze 2004] R. Brown and G. Janelidze, "Galois theory and a new homotopy double groupoid of a map of spaces", Appl. Categ. Structures 12:1 (2004), 63-80. MR 2005e:55016 Zbl 1050.18005

[Brown and Mackenzie 1992] R. Brown and K. C. H. Mackenzie, "Determination of a double Lie groupoid by its core diagram”, J. Pure Appl. Algebra 80:3 (1992), 237-272. MR 93g:55022 Zbl 0766.22001

[Brown and Spencer 1976] R. Brown and C. B. Spencer, "Double groupoids and crossed modules", Cahiers Topologie Géom. Différentielle 17:4 (1976), 343-362. MR 55 \#13427 Zbl 0344.18004

[Dragulete 2007] O. M. Dragulete, Some applications of symmetries in differential geometry and dynamical systems, thesis, Ècole Polytechnique Fèdèrale de Lausanne, 2007, Available at http:// library.epfl.ch/en/theses?nr=3937.

[Ehresmann 1963] C. Ehresmann, "Catégories doubles et catégories structurées", C. R. Acad. Sci. Paris 256 (1963), 1198-1201. MR 27 \#2539 Zbl 0132.25702

[Higgins 2005] P. J. Higgins, "Categories and groupoids", Repr. Theory Appl. Categ. 7 (2005), 1195. MR 2005k:20137 Zbl 1087.20038

[Lang 2002] S. Lang, Introduction to differentiable manifolds, 2nd ed., Springer, New York, 2002. MR 2003h:58002 Zbl 1008.57001

[Lu and Weinstein 1989] J.-H. Lu and A. Weinstein, "Groupoïdes symplectiques doubles des groupes de Lie-Poisson”, C. R. Acad. Sci. Paris Sér. I Math. 309:18 (1989), 951-954. MR 91i:58045 Zbl 0701.58025

[Mackenzie 1992] K. C. H. Mackenzie, "Double Lie algebroids and second-order geometry, I", Adv. Math. 94:2 (1992), 180-239. MR 93f:58255 Zbl 0765.57025

[Mackenzie 1999] K. C. H. Mackenzie, "On symplectic double groupoids and the duality of Poisson groupoids”, Internat. J. Math. 10:4 (1999), 435-456. MR 2000g:58029 Zbl 0961.58008

[Mackenzie 2000] K. C. H. Mackenzie, "Double Lie algebroids and second-order geometry, II", Adv. Math. 154:1 (2000), 46-75. MR 2002f:58025 Zbl 0971.58015 
[Mackenzie 2005] K. C. H. Mackenzie, General theory of Lie groupoids and Lie algebroids, London Mathematical Society Lecture Note Series 213, Cambridge University Press, Cambridge, 2005. MR 2006k:58035 Zbl 1078.58011

[Pradines 1977] J. Pradines, Fibrés vectoriels doubles et calcul des jets non holonomes, Esquisses Mathématiques 29, Université d'Amiens U.E.R. de Mathématiques, Amiens, 1977. MR 83b:58010 Zbl 0396.53016

[Tu 2004] J.-L. Tu, "Non-Hausdorff groupoids, proper actions and $K$-theory", Doc. Math. 9 (2004), 565-597. MR 2005h:22004 Zbl 1058.22005 arXiv math.OA/0403071

Received March 7, 2011. Revised September 13, 2011.

NiCOLAS ANDRUSKIEWITSCH

FACULTAd DE MATEMÁTICAS ASTRONOMÍA Y Física

UNIVERSIDAD NACIONAL DE CÓRDOBA

FAMAF-CIEM (CONICET)

Medina Allende S/N, CiUdad Universitaria

5000 CÓRDOBA

ARGENTINA

andrus@famaf.unc.edu.ar

http://www.famaf.unc.edu.ar/ andrus/

JESUS OCHOA ARANGO

DEPARTAMENTO DE MATEMÁTICAS

PONTIFICIA UNIVERSIDAD JAVERIANA

Cra. 7 No. 43-82 - Edificio CARLos Ortíz

BOGOTÁ

COLOMBia

jesus.ochoa@javeriana.edu.co

AlEJANDRo TIRABOSCHI

FACULTAD DE MATEMÁTICAS ASTRONOMÍA Y FíSICA

UNIVERSIDAD NACIONAL DE CÓRDOBA

FAMAF-CIEM (CONICET)

Medina Allende S/N, CiUdad Universitaria

5000 CÓRDOBA

ARGENTINA

tirabo@famaf.unc.edu.ar 


\title{
PACIFIC JOURNAL OF MATHEMATICS
}

\author{
http://pacificmath.org \\ Founded in 1951 by \\ E. F. Beckenbach (1906-1982) and F. Wolf (1904-1989)
}

\section{EDITORS}

V. S. Varadarajan (Managing Editor)

Department of Mathematics

University of California

Los Angeles, CA 90095-1555

pacific@math.ucla.edu

Vyjayanthi Chari

Department of Mathematics

University of California

Riverside, CA 92521-0135

chari@math.ucr.edu

\section{Robert Finn}

Department of Mathematics Stanford University

Stanford, CA 94305-2125

finn@math.stanford.edu

Kefeng Liu

Department of Mathematics

University of California

Los Angeles, CA 90095-1555

liu@math.ucla.edu
Darren Long

Department of Mathematics

University of California

Santa Barbara, CA 93106-3080

long@math.ucsb.edu

Jiang-Hua Lu

Department of Mathematics

The University of Hong Kong

Pokfulam Rd., Hong Kong jhlu@maths.hku.hk

Alexander Merkurjev

Department of Mathematics

University of California

Los Angeles, CA 90095-1555

merkurev@math.ucla.edu
Sorin Popa

Department of Mathematics University of California

Los Angeles, CA 90095-1555 popa@math.ucla.edu

Jie Qing

Department of Mathematics

University of California

Santa Cruz, CA 95064

qing@cats.ucsc.edu

Jonathan Rogawski

Department of Mathematics

University of California

Los Angeles, CA 90095-1555

jonr@math.ucla.edu

\section{PRODUCTION}

pacific@math.berkeley.edu

\section{SUPPORTING INSTITUTIONS}

ACADEMIA SINICA, TAIPEI

CALIFORNIA INST. OF TECHNOLOGY INST. DE MATEMÁTICA PURA E APLICADA KEIO UNIVERSITY

MATH. SCIENCES RESEARCH INSTITUTE NEW MEXICO STATE UNIV.

OREGON STATE UNIV.

\author{
STANFORD UNIVERSITY \\ UNIV. OF BRITISH COLUMBIA \\ UNIV. OF CALIFORNIA, BERKELEY \\ UNIV. OF CALIFORNIA, DAVIS \\ UNIV. OF CALIFORNIA, LOS ANGELES \\ UNIV. OF CALIFORNIA, RIVERSIDE \\ UNIV. OF CALIFORNIA, SAN DIEGO \\ UNIV. OF CALIF., SANTA BARBARA
}

\author{
UNIV. OF CALIF., SANTA CRUZ \\ UNIV. OF MONTANA \\ UNIV. OF OREGON \\ UNIV. OF SOUTHERN CALIFORNIA \\ UNIV. OF UTAH \\ UNIV. OF WASHINGTON \\ WASHINGTON STATE UNIVERSITY
}

These supporting institutions contribute to the cost of publication of this Journal, but they are not owners or publishers and have no responsibility for its contents or policies.

See inside back cover or pacificmath.org for submission instructions.

The subscription price for 2012 is US \$420/year for the electronic version, and \$485/year for print and electronic.

Subscriptions, requests for back issues from the last three years and changes of subscribers address should be sent to Pacific Journal of Mathematics, P.O. Box 4163, Berkeley, CA 94704-0163, U.S.A. Prior back issues are obtainable from Periodicals Service Company, 11 Main Street, Germantown, NY 12526-5635. The Pacific Journal of Mathematics is indexed by Mathematical Reviews, Zentralblatt MATH, PASCAL CNRS Index, Referativnyi Zhurnal, Current Mathematical Publications and the Science Citation Index.

The Pacific Journal of Mathematics (ISSN 0030-8730) at the University of California, c/o Department of Mathematics, 969 Evans Hall, Berkeley, CA 94720-3840, is published monthly except July and August. Periodical rate postage paid at Berkeley, CA 94704, and additional mailing offices. POSTMASTER: send address changes to Pacific Journal of Mathematics, P.O. Box 4163, Berkeley, CA 94704-0163.

PJM peer review and production are managed by EditFLOW ${ }^{\mathrm{TM}}$ from Mathematical Sciences Publishers.

PUBLISHED BY PACIFIC JOURNAL OF MATHEMATICS

at the University of California, Berkeley 94720-3840

A NON-PROFIT CORPORATION

Typeset in LATEX

Copyright $(02012$ by Pacific Journal of Mathematics 


\section{PACIFIC JOURNAL OF MATHEMATICS}

Volume $256 \quad$ No. $1 \quad$ March 2012

On slim double Lie groupoids

NiCOlas ANDruskiewitsch, JEsus OCHOA ARANGO and AlEJANDRO

TIRABOSCHI

Topological classification of quasitoric manifolds with second Betti number 2

19

Suyoung Choi, SeOnjeOng PARK and Dong Youp SuH

Refined Kato inequalities for harmonic fields on Kähler manifolds

DANiEL Cibotaru and PENG ZHU

Deformation retracts to the fat diagonal and applications to the existence of peak solutions of nonlinear elliptic equations

E. Norman Dancer, Jonathan Hillman and Angela Pistoia

Descent for differential Galois theory of difference equations: confluence and $q$-dependence

LuCia Di Vizio and Charlotte HaRdouin

Modulation and natural valued quiver of an algebra

FANG LI

Willmore hypersurfaces with two distinct principal curvatures in $\mathbb{R}^{n+1}$

TONGZHU LI

Variational inequality for conditional pressure on a Borel subset

YuAN Li, ERCAI CHEN and WEN-ChIAO CHENG

New homotopy 4-spheres

DANIEL NASH

Combinatorial constructions of three-dimensional small covers

YASUZO NISHIMURA

On a theorem of Paul Yang on negatively pinched bisectional curvature

AERYEONG SEO

Orders of elements in finite quotients of Kleinian groups

PETER B. Shalen

A new algorithm for finding an 1.c.r. set in certain two-sided cells

JIAN-YI SHI

Addendum to the article Superconnections and parallel transport

FLORIN DUMITRESCU 